\title{
Efficacy and tolerance of 5 -aminosalicylic acid suppositories in the treatment of ulcerative proctitis: A review of two double-blind, placebo controlled trials
}

CN WiLliams, MD, FRCPC, FACP, FACG

\begin{abstract}
The efficacy and tolerance of $500 \mathrm{mg}$ 5-aminosalicylic acid (mesalamine) suppositories in the treatment of ulcerative proctitis were assessed in two double-blind, placebo controlled studies of six weeks' duration, involving a total of 173 patients. In trial 1 , patients used one $500 \mathrm{mg}$ suppository tid. In trial 2, patients used one $500 \mathrm{mg}$ suppository bid. Physician's global assessment and a disease activity index based upon patient symptoms and sigmoidoscopic appearance were used to assess efficacy. At the endpoint of trial 1 (tid), there was an $80.4 \%$ mean reduction in the disease activity index seen in patients treated with mesalamine compared to a $36.8 \%$ mean reduction in the placebo group $(\mathrm{P}<0.05)$. Analysis of the physician's global assessment indicated that $84.2 \%$ of patients receiving mesalamine were considered to be 'much improved' compared to $41 \%$ of patients on placebo $(\mathrm{P}<0.01)$. At the endpoint of trial 2 (bid), there was a $74.7 \%$ mean reduction in disease activity index compared to $34.2 \%$ in the placebo group $(P<0.001)$. Analysis of the physician's global assessment indicated that $79.2 \%$ of the mesalamine group was considered to be 'much improved' compared to $26.2 \%$ on placebo ( $\mathrm{P}<0.001$ ). There was no significant difference in efficacy seen in patients treated with $500 \mathrm{mg}$ mesalamine suppositories bid or tid. Mesalamine suppositories are effective and well tolerated in the treatment of ulcerative proctitis. Can J Gastroenterol 1990;4(7):472-475 (pour résumé, voir page 473)
\end{abstract}

\section{Key Words: 5-Aminosalicylic acid, Mesalamine, Ulcerative proctitis}

Division of Gastroenterology, Department of Medicine, Dalhousie University, Halifax, Nova Scotia

Correspondence and reprints: Dr CN Williams, Professor and Head, Division of Gastroenterology, Dalhousie University Clinical Research Centre, Room CD-1, 5849 University Avenue, Halifax, Nova Scotia B3H $4 \mathrm{H} 7$
U LCERATIVE PROCTITIS, A MILDER variant of idiopathic ulcerative colitis, represents approximately onethird of the ulcerative colitis population. Mucosal inflammation is limited to the rectum with pathologic changes similar to those seen in ulcerative colitis which often remain quite superficial. Rectal bleeding is the usual presenting symptom and is often accompanied by tenesmus and changes in bowel habits.

In the past, the medical management of ulcerative proctitis has been confined to treatment with sulphasalazine, corticosteroids (topical and oral) and, more recently, 5-aminosalicylic acid (5-ASA, mesalamine) enemas. It is known that sulphasalazine consists of two molecules linked by a diazo bond: 5-ASA and sulfapyridine (1). Studies have shown that 5-ASA is the active moiety, and that many of the side effects which have limited the usefulness of sulphasalazine, can be attributed to the sulpha portion $(2,3)$. 
Efficacité et innocuité des suppositoires à base d'acide

\section{5-aminosalicylique dans le traitement de la rectite ulcéreuse:} Revue de deux essais à double insu contrôlés contre placebo

RESUME: On a effectué deux essais à double insu et contrôlés contre placebo afin d'évaluer l'efficacité et l'innocuité des suppositoires (mésalamine) contenant $500 \mathrm{mg}$ d'acide aminosalicylique dans le traitement de la rectite ulcéreuse. Cette étude de six semaines portaient sur 173 patients. Dans le premier essai, les patients ont reçu un suppositoire de $500 \mathrm{mg}$ trois fois par jour; et dans le second, un suppositoire de $500 \mathrm{mg}$ deux fois par jour. L'efficacité a été déterminée en fonction du bilan général du médecin et de l'indice d'activité de la maladie (DAI) évaluant les symptômes du patients et l'aspect sigmoïdoscopique. A la fin de l'essai $1\left(3 x_{j}\right)$, on a noté une réduction moyenne de $80,4 \%$ dans le DAI des patients sous mésalamine par rapport à une réduction moyenne de $36,8 \%$ dans le groupe placebo $(P<0,05)$. L'analyse du bilan général du médecin indique «une amélioration considérable» pour $84,2 \%$ des patients traités par mésalamine, par rapport à $41 \%$ dans le groupe placebo $(\mathrm{P}<0,01)$. A la fin de l'essai II $\left(2 x_{j}\right)$, on a noté une réduction moyenne de $74,7 \%$ dans le DAI des patients sous mésalamine par rapport à une réduction moyenne de $34,2 \%$ dans le groupe placebo $(\mathrm{P}<0,001)$. L'analyse du bilan général du médecin indique «une amélioration considérable» pour $79,2 \%$ des patients sous mésalamine, par rapport à $26,2 \%$ dans le groupe placebo $(\mathrm{P}<0,001)$. On n'a noté aucune différence significative entre les patients recevant $500 \mathrm{mg}$ de mésalamine deux fois ou trois fois par jour. Les suppositoires de mésalamine sont efficaces et bien tolérés dans le traitement de la rectite ulcéreuse.
Various studies have demonstrated the efficacy of 5-ASA administered as a rectal suspension enema in the treatment of distal ulcerative colitis and proctitis (4-7).

The purpose of these two studies was to evaluate the efficacy and tolerance of a $500 \mathrm{mg}$ mesalamine suppository (Rowasa; Reid-Rowell Inc, Georgia) in the treatment of ulcerative proctitis.

\section{MATERIALS AND METHODS}

Both trials used a six week, randomized, double-blind, parallel, placebo controlled, multicentre design. The protocol was reviewed by the individual ethics committees or internal review boards of the participating institutions. Written informed consent was obtained from each patient before entrance into the study. The study population was composed of outpatients over 18 years old who had ulcerative proctitis (confirmed by sigmoidoscopy) involving a maximum of $15 \mathrm{~cm}$ measured from the anal margin, and a score of three on the 12 point disease activity index. Patients who had a history of salicylate allergy, positive stool culture, clinically significant hepatic or renal disease or pregnancy, or who were taking other rectal medications within two weeks prior to enrollment in the study were excluded. Patients were randomized to receive either placebo or mesalamine suppositories. In trial 1, patients used one $500 \mathrm{mg}$ suppository three times a day. In trial 2, patients used one $500 \mathrm{mg}$ suppository twice daily.

Concurrent medications: Oral steroids or sulphasalazine were allowed in both studies, provided they had been used for at least three weeks prior to entry and that the dose was held constant during the trial. Concomitant use of other rectal medications was not permitted.

Disease activity index: The disease activity index (Table 1) provides objective criteria for evaluating drug efficacy. Four variables were assessed: stool frequency, rectal bleeding, sigmoidoscopic appearance of mucosa and physician's

\section{TABLE 1}

Disease activity index (qualitative rating scale with four subscales)

\begin{tabular}{cl}
\hline Stool frequency & Normal \\
0 & $1-2$ stools/day $>$ normal \\
2 & $3-4$ stools/day $>$ normal \\
3 & $>4$ stools/day $>$ normal \\
Rectal bleeding \\
0 & None \\
1 & Streaks of blood \\
2 & Obvious blood \\
3 & Mostly blood \\
Mucosal appearance \\
$0 \quad$ Normal \\
1 & Mild friability \\
2 & Moderate friability \\
3 & Exudation, spontaneous bleeding \\
Physician's rating of disease severity \\
0 & Normal \\
1 & Mild \\
2 & Moderate \\
3 & Severe \\
\hline Maximumscore 12
\end{tabular}

Maximum score 12

overall assessment of disease severity (8). Each variable has a range of values for disease severity, with ' $O$ ' representing no abnormality and ' 3 ' representing the most severe abnormality.

Procedures: On entry into both studies, a complete history, physical examination and flexible sigmoidoscopy were performed. Hematologic complete blood count and biochemical parameters including urinalysis were assessed along with stool cultures to exclude infectious colitis. In addition, the disease activity index was calculated. Patients were provided with a diary for the daily recording of number of stools, rectal bleeding and other symptoms as well as possible side effects. At week 3, repeat sigmoidoscopy was performed and the disease activity index reassessed. Upon conclusion of the study at week 6 , a repeat history, physical examination, sigmoidoscopy and hematologic and biochemical assessments were carried out. The disease

\section{TABLE 2}

Patient characteristics in two trials of 5-aminosalicylic acid (5-ASA) in ulcerative proctitis

\begin{tabular}{lllll}
\hline & \multicolumn{2}{c}{ Trial 1 (tid) } & \multicolumn{2}{c}{ Trial 2 (bid) } \\
& 5-ASA & Placebo & 5-ASA & Placebo \\
\hline Number of patients & 39 & 40 & 50 & 44 \\
Sex ratio (male/female) & $16 / 23$ & $18 / 22$ & $18 / 32$ & $18 / 26$ \\
Mean age (years) & 35.9 & 41.9 & 37.0 & 40.2 \\
Mean upper disease boundary $(\mathrm{cm})$ & 10.8 & 10.9 & 10.5 & 10.1 \\
\hline
\end{tabular}


TABLE 3

Disease activity index: Percentage of patients assessed 'normal' at endpoint of trial 1 (5-aminosalicylic acid (5-ASA) tid)

\begin{tabular}{lccc}
\hline & 5-ASA & Placebo & P \\
\hline Normal stool frequency & 78.4 & 30.0 & $<0.01$ \\
No rectal bleeding & 89.2 & 42.5 & $<0.001$ \\
Normal mucosal appearance & 62.2 & 25.0 & $<0.001$ \\
Normal physician's rating of disease severity & 62.2 & 22.5 & $<0.001$ \\
\hline
\end{tabular}

TABLE 4

Disease activity index: Percentage of patients assessed 'normal' at endpoint of trial 2 (5-aminosalicylic acid (5-ASA) bid)

\begin{tabular}{lccc}
\hline & 5-ASA & Placebo & P \\
\hline Normal stool frequency & 70.8 & 35.7 & $<0.01$ \\
No rectal bleeding & 68.8 & 16.7 & $<0.001$ \\
Normal mucosal appearance & 60.4 & 9.8 & $<0.001$ \\
Normal physician's rating of disease severity & 60.4 & 9.5 & $<0.001$ \\
\hline
\end{tabular}

activity index was again calculated.

Statistical analysis: Nonparametric data ('physician's global assessment') were analyzed by $\chi^{2}$ tests. Changes in disease activity index (an ordinal scale) were analyzed by analysis of variance.

\section{RESULTS}

Patient population: Seventy-nine patients entered trial 1 (tid) and 94 patients entered trial 2 (bid). In both studies, active and placebo groups were comparable with regard to sex, age and disease boundary (Table 2). Twentyseven patients ( 10 on active medication and 17 on placebo) withdrew from the studies -10 from trial 1 and 17 from trial 2. Of the 10 mesalamine-treated patients who withdrew, three withdrew due to adverse experiences and one due to lack of therapeutic effectiveness.

Response to therapy: In both trials, response to therapy was assessed by changes in the overall disease activity index as well as in the individual disease activity index parameters. Additionally, at the conclusion of the treatment period, the physician provided a final global assessment reflecting the degree of improvement.

Trial 1 (tid): At the end of six weeks, the mesalamine-treated patients had an $80.4 \%$ mean reduction in overall disease activity index, which was significantly greater $(P<0.05)$ than the mean reduction of $36.8 \%$ seen in the placebo group.
Each of the disease activity index parameters showed a highly significant difference between groups in the percentage of patients assessed to be 'normal', ie, score of 0 at the endpoint (Table 3).

In addition, $84.2 \%$ (32 of 38 ) of mesalamine patients were considered 'much improved' by the physician's global assessment compared to $41 \%$ (16 of 39) of placebo patients $(\mathrm{P}<0.01)$.

Trial 2 (bid): The mean reduction in the overall disease activity index score was $74.7 \%$ in the mesalamine-treated patients and $34.2 \%$ in the placebotreated patients $(P<0.001)$. Each of the disease activity index parameters demonstrated a significant difference in the percentage of patients assessed to be 'normal' at the endpoint (Table 4).

The physician's final global assessment showed that $79.2 \%$ of sidered 'much improved' compared to $26.2 \%$ of placebo-treated patients $(\mathrm{P}<0.001)$.

There was no statistically significant difference in efficacy seen between patients treated with $500 \mathrm{mg}$ mesalamine three times daily (trial 1) and those treated with $500 \mathrm{mg}$ mesalamine twice daily (trial 2).

Side effects: Adverse effects were few and insignificant in both trials. The incidence was similar in the mesalamine and placebo groups with the most frequently reported adverse experience in mesalamine-treated patients were con- both groups being headache (11\%), flatulence $(7 \%)$, diarrhea $(6 \%)$ and abdominal pain $(6 \%)$. Only three of the 89 mesalamine-treated patients discontinued treatment due to an adverse experience. These events included one case of skin rash, one case of rectal irritation, and one patient who experienced an increase in diarrhea and mucosal inflammation while using the suppositories.

\section{DISCUSSION}

The therapeutic options available in the treatment of ulcerative proctocolitis have previously relied upon the use of sulphasalazine and various corticosteroid preparations. However, significant side effects can be attributed to either treatment. Clinical experience with 5-ASA enemas in addition to these modalities have proven them to be effective and well tolerated in the treatment of left-sided ulcerative colitis (4-7).

This report on the use of the suppository form of mesalamine in the treatment of ulcerative proctitis demonstrated a significant improve. ment in each measure of disease activity.

In both studies, the mesalamine group demonstrated a significant mean reduction in the disease activity index compared to placebo. In addition, each of the individual parameters comprising the disease activity index demonstrated a significantly greater remission rate, ie, score of 0 , compared to placebo. Finally, the physician's global assessment of patients at the end of the study showed a significant im. provement. There was no statistical difference in efficacy between patients treated with $500 \mathrm{mg}$ mesalamine bid versus tid.

Few patients reported adverse side effects, and those that were reported were not of a serious nature.

\section{CONCLUSIONS}

Mesalamine $500 \mathrm{mg}$ suppositories, administered bid or tid, are effective and well tolerated in the treatment of ulcerative proctitis and may offer advantages over existing therapeutic modalities. 
ACKNOWLEDGEMENTS: The author is grateful to the following investigators who participated in the study: Kim Culp, MD of Temple, Texas; Larry Good, MD of Merrick, New York; Gregory Haber, MD of Toronto, Ontario; Stephen Hanauer, MD of Chicago, Illinois; Indru Khubchandani, MD of Allentown, Pennsylvania; Carl Lyss, MD of St Louis, Missouri; Gerald Onstad, MD of Minneapolis, Minnesota; Ronald Pizinger, MD of St Paul, Minnesota; Malcolm Robinson, MD of Oklahoma City, Oklahoma; and Williamson Strum, MD of Lajolla, California. In addition, the author would like to thank Lowell Borgen, PhD for his technical assistance with the study and David Rothbart, MD, for his help and advice in preparation of the manuscript. This work was supported by a grant from Reid-Rowell Inc of Marietta, Georgia.

\section{REFERENCES}

1. Svartz N. Salazopyrin, a new sulfanilamide preparation. Acta Med Scand 1942;110:577-98.

2. Peppercorn MA, Goldman P. Distribution studies of salicylazosulfapyridine and its metabolites. Gastroenterology 1973;64:240-55.

3. Azad Khan KA, Piris T, Truelove SC. An experiment to determine the active therapeutic moiety of sulphasalazine. Lancet 1973;ii:892-5.

4. McPhee MS, Swan JT, Biddle WL, Greenberger NJ. Proctocolitis unresponsive to conventional therapy: Response to 5-ASA enemas. Dig Dis Sci 1987;32:765-815.
5. Sutherland LR, Martin F, Greer S, et al. 5-ASA enema in the treatment of distal ulcerative colitis, proctosigmoiditis and proctitis. Gastroenterology 1987;92:1894-8.

6. Campieri M, Gionchetti P, Belluzi A, et al. Efficacy of 5-ASA enemas versus hydrocortisone enemas in ulcerative colitis. Dig Dis Sci 1987;32:675-705.

7. Guarino J, Chatzinoff M, Berk T, et al. 5-ASA enemas in refractory distal ulcerative colitis: Long term results. Am J Gastroenterol 1987;82:732-7.

8. Powell-Tuck J, Day DW, Buckell NA, et al. Correlations between sigmoidoscopic appearance and other measures of disease activity in ulcerative colitis. Dig Dis Sci 1982;27:533-7. 


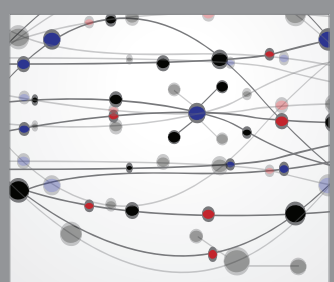

The Scientific World Journal
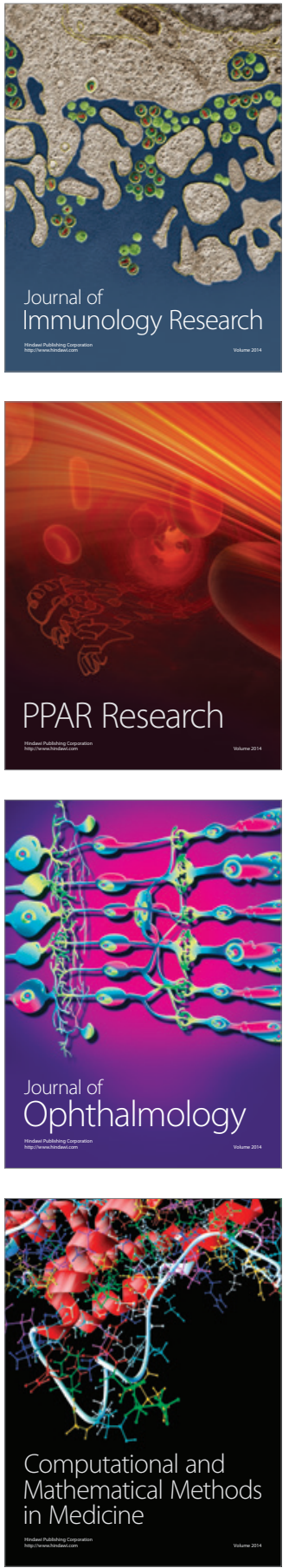

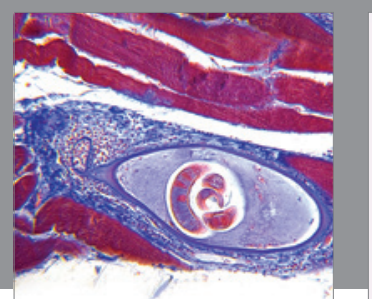

Gastroenterology Research and Practice

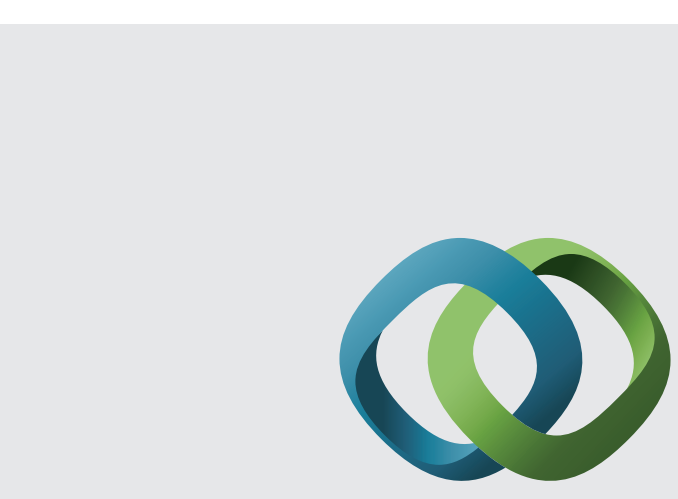

\section{Hindawi}

Submit your manuscripts at

http://www.hindawi.com
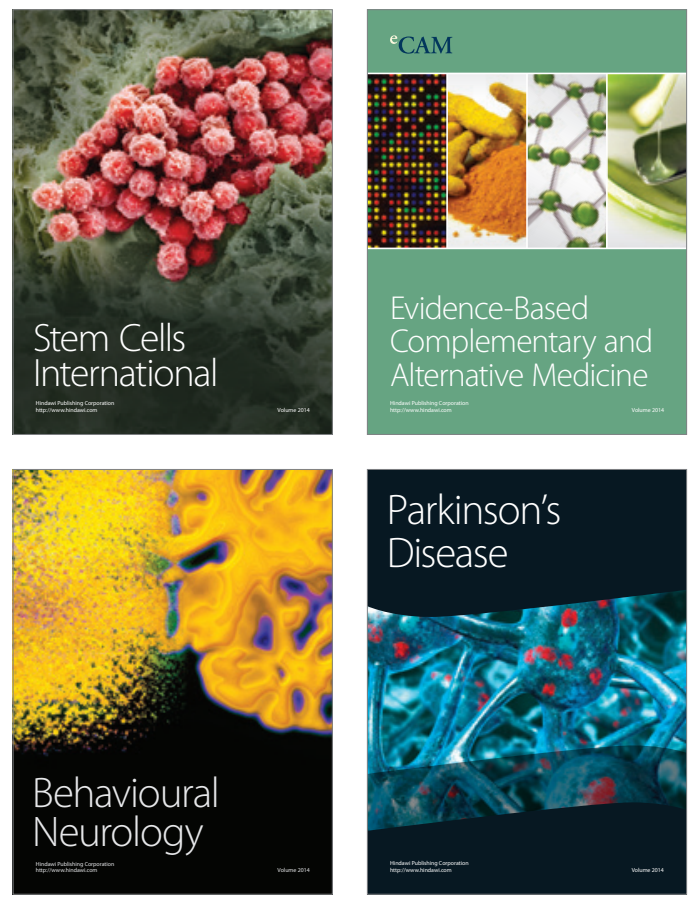
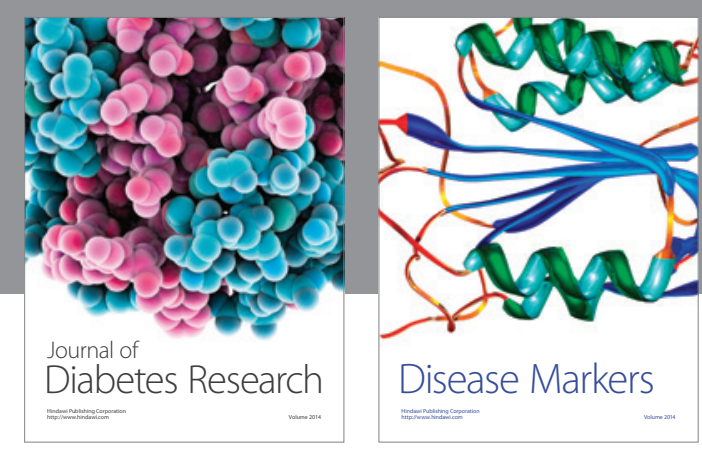

Disease Markers
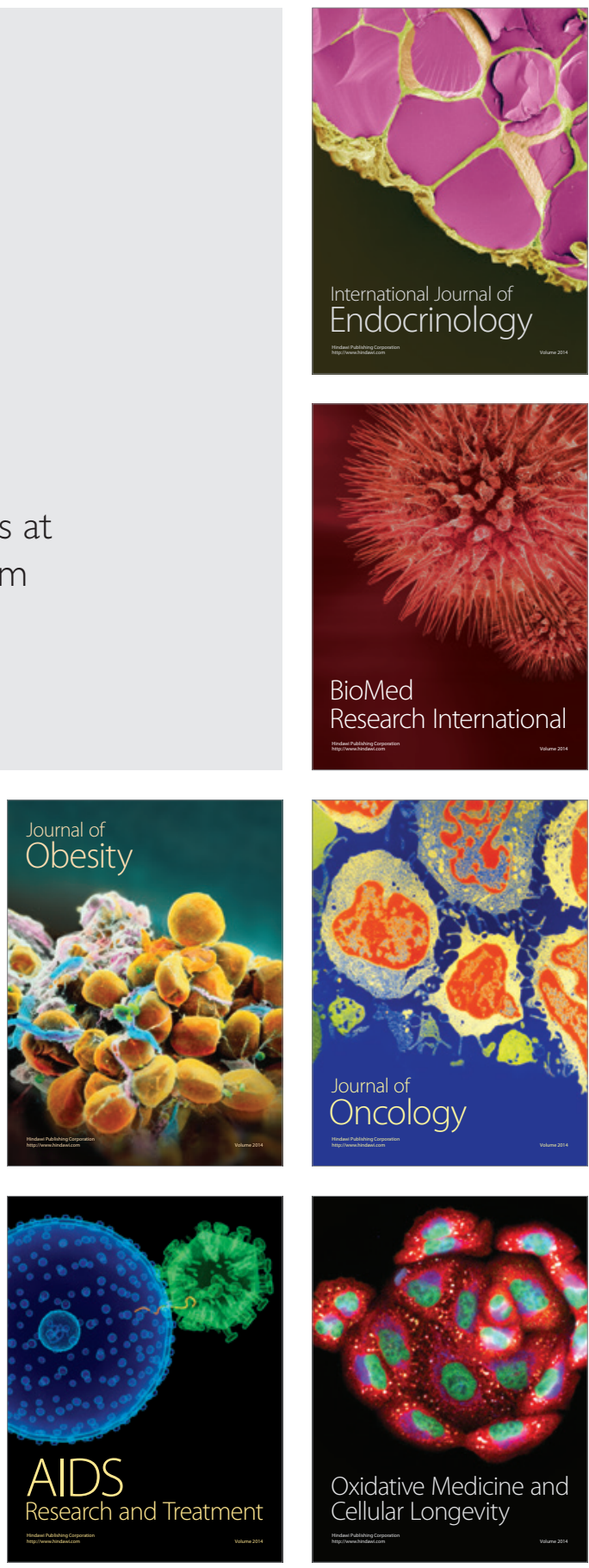